\title{
Cut-on cut-off transition in flow ducts: comparing multiple-scales and finite-element solutions
}

\author{
Nick C. Ovenden* \\ University College London, WC1E 6BT, United Kingdom. \\ Walter Eversman ${ }^{\dagger}$ \\ University of Missouri-Rolla, Rolla, MO 65409-0257, USA. \\ Sjoerd W. Rienstra \\ Eindhoven University of Technology, 5600 MB Eindhoven, The Netherlands.
}

\begin{abstract}
The phenomenon of cut-on cut-off transition of acoustic modes in ducts with mean flow is examined using an analytical multiple-scales solution, and compared to solutions obtained from a numerical finite-element method. The analytical solution, derived for an arbitrary duct with irrotational mean flow, remains valid to leading-order throughout the duct. In other words, it is a composite solution, encompassing both the inner boundary-layer solution in the neighbourhood of the transition point and the outer slowly varying modal solution far upstream and downstream. Several test cases are defined and presented within a geometry representative of a high-bypass turbofan engine. The cases span a wide realistic range of frequencies and circumferential mode numbers both with and without mean flow, including one numerically-challenging investigation of cutoff cut-on transition of a mode. The agreement is in most cases remarkably good. Slight differences in position of the pressure pattern can be observed for cases with mean flow, which seem due to the slight variations in mean flow fields obtained from both methods. When cut-on cut-off transition occurs for high Helmholtz number and high radial mode number a certain amount of modal scattering is observed. An attempt is made to explain this by incorporating the presence of neighbouring modes in the asymptotic scaling arguments for the turning point region. The composite solution should enable designers to continue to use multiple-scales theory to examine flow pressure and noise transmission inside an engine duct, whilst now being able to include directly the contributions of modes undergoing transition without encountering singular behaviour.
\end{abstract}

\section{Nomenclature}

$\begin{array}{ll}\mathrm{Ai}, \mathrm{Bi} & =\text { Airy functions } \\ E & =\text { mean flow Bernoulli constant } \\ F & =\text { mean flow mass flux } \\ \mathrm{J}_{m}, \mathrm{Y}_{m} & =\text { Bessel functions of the first and second kinds of order } m \\ m & =\text { circumferential modal wave number } \\ M & =\text { Mach number } \\ n & =\text { radial modal order } \\ N & =\text { slowly varying modal amplitude } \\ n_{1}, n_{2} & =\text { unit outer normal vectors of } R_{1}, R_{2} \\ Q & =\text { constant to fix modal amplitude } \\ \bar{Q} & =\text { related to } Q, \text { see Eq. }(22) \\ R_{1}, R_{2} & =\text { inner, outer walls of duct }\end{array}$

\footnotetext{
${ }^{*}$ Research Fellow, Department of Mathematics, University College London, Gower Street, London WC1E 6BT, United Kingdom. AIAA Member.

†Curators' Professor, Department of Mechanical and Aerospace Engineering and Engineering Mechanics, University of Missouri-Rolla, 1870 Miner Circle, Rolla, MO 65409-0257, USA. AIAA Member.

$¥$ Associate Professor, Department of Mathematics and Computer Science, Eindhoven University of Technology, P.O. Box 513, 5600 MB Eindhoven, The Netherlands. AIAA Member.
} 


$\begin{array}{ll}R_{\infty}, \rho_{\infty}, c_{\infty} & =\text { reference values, } \\ \tilde{\boldsymbol{v}}, \tilde{p}, \tilde{\rho}, \tilde{c}, \tilde{\phi} & =\text { velocity, pressure, density, sound speed, potential } \\ \boldsymbol{v}, p, \rho, c, \phi & =\text { time-harmonic velocity, pressure, density, sound speed, potential perturbations } \\ \boldsymbol{V}, P, D, C & =\text { mean flow velocity, pressure, density, sound speed } \\ U_{0}, \boldsymbol{V}_{\perp 0}, P_{0}, D_{0}, C_{0} & =\text { approximate mean flow variables } \\ x, r, \theta, t & =\text { axial, radial, azimuthal angle, time coordinate } \\ \boldsymbol{e}_{x}, \boldsymbol{e}_{r}, \boldsymbol{e}_{\theta} & =\text { unit vectors in } x, r, \theta \text {-direction } \\ X & =\varepsilon x, \text { slow variable } \\ X_{t}, x_{t} & =\text { cut-on cut-off transition point in } X, x \text { coordinate } \\ \alpha & =\text { (square root of) eigenvalue of } \psi ; \text { in axisymmetric geometry: radial modal wave number } \\ \gamma & =\text { ratio of specific heats } \\ \varepsilon & =\text { small parameter, representing typical axial duct slope } \\ \zeta & =\frac{2}{3}|x|^{3 / 2} \\ \mu & =\text { axial modal wave number } \\ \sigma & =\text { reduced axial wave number, Eq. (19) } \\ \Upsilon & =\text { factor of } Y_{m} \text { in annular duct eigensolution } \\ \psi & =\text { eigensolution of Laplace operator in a cross-sectional plane } \\ \omega & =\text { Helmholtz number (dimensionless angular frequency) }\end{array}$

\section{Introduction}

SOUND transmission through a duct of slowly-varying cross section can be modelled using the theory of multiple $S_{\text {scales. This theory was first applied to ducts without mean flow }}{ }^{1}$ and extended in later works to include cases with mean irrotational flow ${ }^{2}$, mean swirling flow ${ }^{3}$ and most recently non-axisymmetric ducts (with mean irrotational flow) ${ }^{4}$ The multiple scales approach provides an attractive alternative to a full numerical solution of acoustic modes in aeroengine ducts, as the calculation complexities are only marginally more than finding the eigenmodes inside a straight duct. Indeed, a recent comparison of multiple-scales solutions of sound propagation with those obtained from a numerical finite-element method shows good agreement across a range of realistic engine frequencies. ${ }^{5}$

The multiple-scales approach allows sound transmission to be represented by a summation of slowly varying modes, which depend on a slow axial variable based on the the slope of the duct walls as a small parameter. Crucially, the amplitudes of these modes vary on the slow scale and are determined via a solvability condition. However, the multiple-scales approximation breaks down at positions where the amplitude of a particular mode becomes singular. These singular transition points are analogous to the turning points observed in solutions to Schrödinger's equation, and represent the complete reflection of a cut-on propagating mode and transmission of a cut-off attenuating mode (or vice-versa).

The turning-point behaviour of such a mode can be analysed by examining the solution in a boundary-layer region encompassing the singularity. Within this region, the original slowly-varying assumption fails to hold and a different approximation leads to the non-convective axial variation of the mode satisfying Airy's equation. Such an analysis was performed for both axisymmetric ${ }^{1,3,6}$ and non-axisymmetric ducts ${ }^{4}$ alike in cases of no mean flow, irrotational mean flow and mean swirling flow (axisymmetric only). In all cases it was shown that the incident cut-on mode is completely reflected in the axial plane with a phase shift of $\pi / 2$. In the event that an isolated acoustic mode undergoes cut-on cut-off transition, no energy is propagated beyond the transition point. Similar partial reflection of modes also appear to occur in lined ducts (so-called near transition) where the reflection coefficient has magnitude and phase determined by the properties of the mean flow and liner impedance. ${ }^{7}$

An understanding of cut-on cut-off behaviour in hard-walled ducts is important for engine design applications. The usual design of rotors and stators is such that at least for the first harmonic all interaction modes are cut-off. Cutting off acoustic modes by varying the duct geometry could, in principle, add to further reduction of the noise output of an engine. However, reflection of a cut-on mode may also result in the mode becoming trapped inside a section of the duct, possibly leading to acoustic resonance and instability; such a scenario has been investigated previously. ${ }^{8}$

In this paper, an explicit analytical solution for transition, either from cut-on to cut-off or vice-versa, is compared against the solution obtained from a numerical finite-element method. The analytical solution ${ }^{9}$, derived for an arbitrary duct with mean irrotational flow, remains uniformly valid to leading-order throughout the duct. In other words, it is a composite solution, encompassing both the inner boundary-layer solution in the neighbourhood of the transition point and the outer slowly varying modal solution far upstream and downstream. This composite solution can therefore be 
applied exactly as a normal slowly-varying mode, without any need to calculate the size of the boundary layer around the transition point, nor match the inner and outer solutions together at some intermediate interface. Such a solution should enable designers to continue to use multiple-scales theory to examine flow pressure and noise transmission inside an engine duct, whilst now being able to include directly the contributions of modes undergoing transition without encountering singular behaviour. The finite-element solution ${ }^{10}$ solves directly the potential flow equations for linear acoustic perturbations of a compressible inviscid isentropic irrotational mean flow, which form the original basis for the multiple scales approximation.

To test the analytical composite solution, absolute pressure plots are compared against those obtained from the finite-element model. Following in a similar manner as a previous comparison of two of the authors ${ }^{5}$, several cases of modes undergoing transition from cut-on to cut-off are examined within a generic engine inlet-duct geometry at realistic engine frequencies, with and without irrotational mean flow. An additional numerically challenging case of an attenuating mode cutting-on in the duct is also examined.

The outline of the paper is as follows. Section II details the derivation of the potential flow governing equations and boundary conditions which are common to both models, along with a description of the duct geometry. The subsequent sections III and IV then describe briefly the derivation of the multiple-scales solution for simple modal transition and the finite-element model respectively. The results and discussion of seven test cases are presented in section V followed by an discussion on the occurrence of modal scattering in section VI and conclusions in section VII.

\section{Potential Flow Model}

\section{A. Derivation of the governing equations}

Consider compressible perfect isentropic irrotational gas flow, consisting of a subsonic mean flow and small acoustic disturbances, inside a duct of slowly-varying cross section. The problem can be non-dimensionalised by scaling all spatial dimensions on a typical duct width $R_{\infty}$, density $\tilde{\rho}$ on some reference value for the gas $\rho_{\infty}$, velocities $\tilde{\boldsymbol{v}}$ and sound speed $\tilde{c}$ on a reference sound speed of the gas $c_{\infty}$, time $t$ on $R_{\infty} / c_{\infty}$ and pressure $\tilde{p}$ on $\rho_{\infty} c_{\infty}^{2}$. The perfect gas condition implies constant heat capacities and the ratio of specific heats at constant pressure and volume is taken as $\gamma=1.4$. It is further assumed that (i) the acoustic variations are too rapid for heat conduction (Péclet number is large) and (ii) the viscous forces are negligible (Reynolds number is large). The resulting governing equations are

$$
\frac{\partial \tilde{\rho}}{\partial t}+\nabla \cdot(\tilde{\rho} \tilde{\boldsymbol{v}})=0, \quad \tilde{\rho}\left(\frac{\partial \tilde{\boldsymbol{v}}}{\partial t}+\tilde{\boldsymbol{v}} \cdot \nabla \tilde{\boldsymbol{v}}\right)+\nabla \tilde{p}=0, \quad \gamma \tilde{p}=\tilde{\rho}^{\gamma}, \quad \tilde{c}^{2}=\frac{\mathrm{d} \tilde{p}}{\mathrm{~d} \tilde{\rho}}=\tilde{\rho}^{\gamma-1}
$$

The assumption that the flow is irrotational allows us to introduce a velocity potential $\tilde{\phi}$, where $\tilde{v}=\nabla \tilde{\phi}$, and to integrate the above momentum equation to obtain a variant of Bernoulli's equation, where

$$
\frac{\partial \tilde{\phi}}{\partial t}+\frac{1}{2}|\nabla \tilde{\phi}|^{2}+\frac{\tilde{c}^{2}}{\gamma-1}
$$

is a conserved quantity throughout the flow.

Following the analysis of Rienstra ${ }^{2}$, the flow is split into a steady irrotational mean flow, with no swirling component, and infinitesimally small harmonic perturbations of angular frequency (Helmholtz number) $\omega>0$ to represent the acoustic part. Thus,

$$
[\tilde{\boldsymbol{v}}, \tilde{\rho}, \tilde{p}, \tilde{c}]=[\boldsymbol{V}, D, P, C]+\operatorname{Re}\left\{[\nabla \phi, \rho, p, c] \mathrm{e}^{\mathrm{i} \omega t}\right\} .
$$

Substitution of this form into the governing equation leads to the following system

$$
\nabla \cdot(D \boldsymbol{V})=0, \quad \frac{1}{2}|\boldsymbol{V}|^{2}+\frac{C^{2}}{\gamma-1}=E, \quad C^{2}=\gamma \frac{P}{D}=D^{\gamma-1},
$$

for the mean flow field, given some constant $E$. The acoustic field can be described, after eliminating pressure and density perturbations, as a solution to the general convected wave equation

$$
\nabla \cdot(D \nabla \phi)-D(\mathrm{i} \omega+\boldsymbol{V} \cdot \nabla)\left[C^{-2}(\mathrm{i} \omega+\boldsymbol{V} \cdot \nabla) \phi\right]=0 .
$$

The pressure and density perturbations can subsequently be recovered from $\phi$ by the expressions

$$
p=-D(\mathrm{i} \omega+\boldsymbol{V} \cdot \nabla) \phi, \quad p=C^{2} \rho .
$$




\section{B. Test geometry and boundary conditions}

Both the multiple-scales solution ${ }^{4,9}$ and the finite-element model ${ }^{10}$ can be applied to ducts of arbitrary shape, although for this paper the inlet duct is taken to be axisymmetric. The duct geometry can be described ideally in cylindrical coordinates $(x, r, \theta)$ where $x$ is the axial coordinate with unit vector $\boldsymbol{e}_{x}, r$ is the radial coordinate with unit vector $\boldsymbol{e}_{r}$ and $\theta$ is the azimuthal coordinate with unit vector $\boldsymbol{e}_{\theta}$. The test geometry chosen represents a realistic generic inlet duct geometry of a high-bypass turbofan engine, similar to the one used in a previous comparison paper of two of the authors. ${ }^{5}$ The positions of the inner spinner and outer nacelle can thus be defined by two nondimensional radial functions $r=R_{1}(x)$ and $r=R_{2}(x)$ respectively. A cross-sectional schematic of the inlet duct used is shown in Fig. i. The reference values for nondimensionalisation are taken at $x=0$, such that $R_{2}(0)=1$. The outer radius $R_{2}$ and inner radius $R_{1}$ are described by the following formulas

$$
\begin{aligned}
& R_{2}(x)=1-0.18453 y^{2}+0.10158 \frac{\mathrm{e}^{-11(1-y)}-\mathrm{e}^{-11}}{1-\mathrm{e}^{-11}}, \\
& R_{1}(x)=\max \left[0,0.64212-\left(0.04777+0.98234 y^{2}\right)^{1 / 2}\right],
\end{aligned}
$$

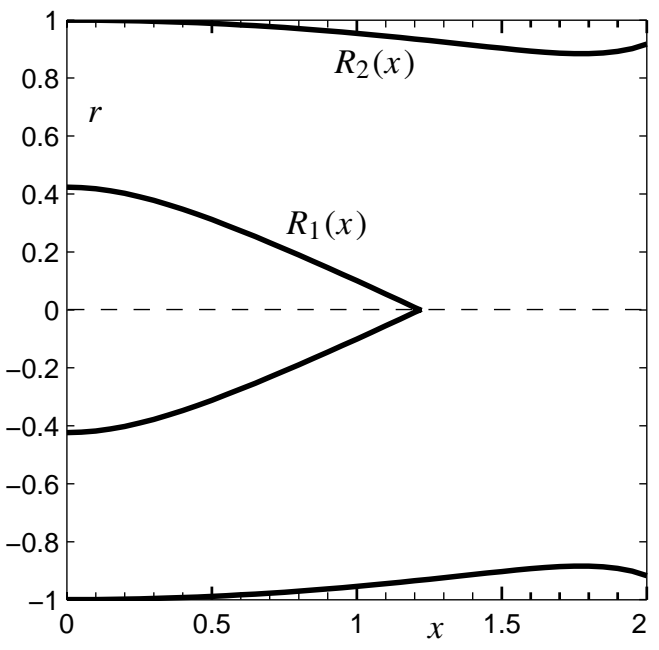

Figure i. Generic inlet duct of a high-bypass turbofan engine.

where $y=x / L$ and $L=2$ (and not 1.86393 like in $^{5}$ ) is the length of the duct.

The sound is taken to be emitted from the left of the source plane at $x=0$, which roughly represents the position of the turbofan inside a real aeroengine. The incident cut-on sound waves then propagate from left-to-right in a positive sense axially. As this is an inlet duct, any mean flow is assumed to come from the opposite direction to the incident sound, approaching the fan from the right of the picture. For both solutions, the mean flow is selected by its Mach number $M$ attained at the source plane $x=0$ with nondimensional sound speed $C$ and density $D$ set equal to unity there (so $U(0)=M$ ). These conditions fix the constant $E$ in the mean flow governing equations, and the axial mass flux $F$. Applying radial boundary conditions that the normal velocity vanishes at the spinner and at the outer wall consequently determines the mean flow field completely throughout the duct.

As we are examining the phenomenon of cut-on cut-off transition, both inner and outer walls are assumed to be hard with infinite impedance. Hence for the acoustic field, the necessary boundary condition to be imposed at the walls is that the normal velocity vanishes at both inner and outer walls. In other words, $\nabla \phi \cdot \boldsymbol{n}_{i}=0$ at $r=R_{i}(x)$, where $\boldsymbol{n}_{1}$ and $\boldsymbol{n}_{2}$ are the outer normals for $R_{1}$ and $R_{2}$ respectively.

\section{Multiple-scales approach and composite solution}

\section{A. Slowly varying mean flow}

The WKB approximation is based on the assumption that the geometry and mean flow are slowly-varying on a length scale much longer than a typical acoustic wave length. As we are interested in waves typically equal to or shorter than a duct radius, geometry and mean flow should vary slowly on a lengthscale much longer than the duct radius. The approximate mean flow solution, compatible with the WKB approach, is then obtained by the method of slow variation. ${ }^{11}$ For the analysis, a slow axial variable $X=\varepsilon x$ is defined, where $\varepsilon \ll 1$ is a small nondimensional parameter representing the typical slope of the duct walls. The slowly varying duct walls can therefore be expressed as functions of this slow variable, so that for any arbitrarily duct $r=R_{1}(X, \theta)$ and $r=R_{2}(X, \theta)$ for inner and outer walls respectively. Note that without change of notation we use in the actual calculations and results the definition from Eqs. (7) of $R_{i}(x)$, i.e. depending on $x$, whereas in the analysis the functions $R_{i}$ will be assumed to depend on $X$.

Assuming the mean flow is irrotational with axial variations in the slow variable $X$ only, it follows ${ }^{4}$ that it is nearly uniform, and we can expand the mean flow variables in terms of $\varepsilon$ to obtain

$$
\begin{aligned}
\boldsymbol{V}(X, r, \theta ; \varepsilon) & =U_{0}(X) \boldsymbol{e}_{x}+\varepsilon \boldsymbol{V}_{\perp 0}(X, r, \theta)+\mathcal{O}\left(\varepsilon^{2}\right), & & P(X, r, \theta ; \varepsilon)=P_{0}(X)+\mathcal{O}\left(\varepsilon^{2}\right), \\
D(X, r, \theta ; \varepsilon) & =D_{0}(X)+\mathcal{O}\left(\varepsilon^{2}\right), & C(X, r, \theta ; \varepsilon) & =C_{0}(X)+\mathcal{O}\left(\varepsilon^{2}\right) .
\end{aligned}
$$

Here, $\varepsilon \boldsymbol{V}_{\perp 0}$ represents a small crosswise mean flow component in the $\boldsymbol{e}_{r}$ and $\boldsymbol{e}_{\theta}$ directions; for the case of an axisymmetric duct, this is purely radial. ${ }^{2}$ The solution to the mean flow equations, Eqs. (4), in terms of the two defined 
physical constants $E$ and $F$ is given by

$$
U_{0}(X)=\frac{F}{D_{0}(X) \int_{0}^{2 \pi} \int_{R_{1}}^{R_{2}} r \mathrm{~d} r \mathrm{~d} \theta}, \quad \frac{1}{2} U_{0}^{2}+\frac{D_{0}^{\gamma-1}}{(\gamma-1)}=E,
$$

with $C_{0}$ and $P_{0}$ obtained from the other relations in Eqs. (4).

\section{B. Slowly varying acoustic modes}

On obtaining a mean flow consistent with the slowly-varying approximation, the method of multiple-scales (MS) enables the acoustic field in a slowly varying duct to be represented as a summation of slowly varying modes of the form $^{4}$

$$
\phi(x, r, \theta ; \varepsilon)=N(X) \psi(r, \theta ; X) \exp \left(-\frac{\mathrm{i}}{\varepsilon} \int^{X} \mu(\zeta ; \varepsilon) \mathrm{d} \zeta\right) .
$$

The function $\psi(r, \theta ; X)$ is the solution to the following eigenvalue problem in the cross-sectional plane

$$
-\left(\frac{1}{r} \frac{\partial}{\partial r}\left(r \frac{\partial}{\partial r}\right)+\frac{1}{r^{2}} \frac{\partial^{2}}{\partial \theta^{2}}\right) \psi=\alpha^{2} \psi
$$

with hard-wall boundary conditions

$$
\frac{\partial \psi}{\partial r}=\frac{1}{R_{i}} \frac{\partial R_{i}}{\partial \theta} \frac{\partial \psi}{\partial \theta} \text { at } r=R_{i}(X, r, \theta), i=1,2
$$

and the slow axial variable $X$ acts as a parameter. The eigenvalue $\alpha^{2}$ with eigensolution $\psi$ satisfies the dispersion relation

$$
\frac{\left(\omega-\mu U_{0}\right)^{2}}{C_{0}^{2}}-\mu^{2}=\alpha^{2}
$$

which, in turn, determines the axial wavenumber $\mu(X ; \varepsilon)=\mu(X)+\mathcal{O}\left(\varepsilon^{2}\right)$. It expedites the analysis ${ }^{4}$ to normalise the eigensolution by integrating its square across the cross-sectional plane at each $X$-station, ensuring

$$
\int_{0}^{2 \pi} \int_{R_{1}(X, \theta)}^{R_{2}(X, \theta)} \psi^{2}(r, \theta ; X) r \mathrm{~d} r \mathrm{~d} \theta=1 .
$$

For our test case of an axisymmetric annular duct, the eigensolution is a combination of Bessel functions of first and second kinds multiplied by $\mathrm{e}^{-\mathrm{i} m \theta}$ for circumferential wavenumber $m$ (for a spinning mode $m \neq 0$ ). Explicitly then, we have ${ }^{9}$

$$
\psi(r, \theta ; X)=\frac{\mathrm{J}_{m}(\alpha r)-\Upsilon(X) \mathrm{Y}_{m}(\alpha r)}{\sqrt{\frac{2}{\pi}\left(\frac{R_{2}{ }^{2}-m^{2} / \alpha^{2}}{\left[\alpha R_{2} \mathrm{Y}_{m}^{\prime}\left(\alpha R_{2}\right)\right]^{2}}-\frac{R_{1}{ }^{2}-m^{2} / \alpha^{2}}{\left[\alpha R_{1} \mathrm{Y}_{m}^{\prime}\left(\alpha R_{1}\right)\right]^{2}}\right)}} \mathrm{e}^{-\mathrm{i} m \theta} \text { for } m \neq 0,
$$

where $\Upsilon(X)$ and the radial eigenvalue $\alpha(X)$ can be determined from the hard-walled boundary condition in Eq. (12) now simplified to $\frac{\partial \psi}{\partial r}=0$. Thus,

$$
\frac{\mathbf{J}_{m}^{\prime}\left[\alpha(X) R_{2}(X)\right]}{\mathrm{Y}_{m}^{\prime}\left[\alpha(X) R_{2}(X)\right]}=\frac{\mathbf{J}_{m}^{\prime}\left[\alpha(X) R_{1}(X)\right]}{\mathrm{Y}_{m}^{\prime}\left[\alpha(X) R_{1}(X)\right]}=\Upsilon(X) .
$$

For a hollow cylindrical duct $\left(R_{1}=0\right)$ these expressions reduce to $\Upsilon(X)=0, \alpha(X)$ determined from the boundary condition $\mathrm{J}_{m}^{\prime}\left(\alpha R_{2}\right)=0$, and

$$
\psi(r, \theta ; X)=\frac{\mathbf{J}_{m}(\alpha r)}{\mathbf{J}_{m}\left(\alpha R_{2}\right)} \sqrt{\frac{2}{\pi}}\left(R_{2}{ }^{2}-\frac{m^{2}}{\alpha^{2}}\right)^{-1 / 2} \mathrm{e}^{-\mathrm{i} m \theta} \text { for } m \neq 0 .
$$

Lastly, the slowly varying amplitude $N(X)$ is determined from a solvability condition ${ }^{2,4,12}$ to be

$$
N(X)=Q \sqrt{\frac{C_{0}(X)}{\omega \sigma(X) D_{0}(X)}},
$$


for some constant $Q$ (obtained from the sound source) and where

$$
\sigma(X)=\sqrt{1-\left(C_{0}^{2}-U_{0}^{2}\right) \frac{\alpha^{2}}{\omega^{2}}}
$$

is defined as the reduced axial wavenumber. ${ }^{2}$ The reduced axial wavenumber is the axial wavenumber rescaled without its convected part, explicitly

$$
\mu=\omega \frac{C_{0} \sigma-U_{0}}{C_{0}^{2}-U_{0}^{2}}
$$

The two solutions of the square root represent two counterpart modes travelling in opposite directions axially along the duct. For cut-on modes that propagate axially in the duct, $\sigma$ is purely real, whereas for cut-off modes that are attenuated and do not propagate along the duct, $\sigma$ is purely imaginary (see Fig. ii).

\section{Cut-on cut-off transition}

Hard-wall transition points occur in a slowly-varying duct when the reduced axial wavenumber, Eq. (19), becomes zero (see Fig. ii) making the modal amplitude $N(X)$ in Eq. (18) singular. Hence in the neighbourhood of such a point $X_{t}$, with $\sigma\left(X_{t}\right)=0$, the slowly varying assumption breaks down and a new approximation to the leading-order governing equations is necessary. For non-swirling mean flow, the analysis performed ${ }^{4,6}$ for an isolated propagating cut-on mode reveals that at the singular point, the mode is completely reflected into its opposite running counterpart with a phase shift of $\frac{1}{2} \pi$. Thus, for such an isolated mode propagating in the positive $X$-direction towards the singular (transitional) point at $X_{t}$ we find that ahead of transition $\left(X<X_{t}\right)$,

$$
\phi=N(X) \psi(r, \theta ; X) \exp \left(\frac{\mathrm{i}}{\varepsilon} \int_{X_{t}}^{X} \frac{\omega U_{0}}{C_{0}^{2}-U_{0}^{2}} \mathrm{~d} X^{\prime}\right)\left[\exp \left(-\frac{\mathrm{i}}{\varepsilon} \int_{X_{t}}^{X} \frac{\omega C_{0} \sigma}{C_{0}^{2}-U_{0}^{2}} \mathrm{~d} X^{\prime}\right)+\mathrm{i} \exp \left(\frac{\mathrm{i}}{\varepsilon} \int_{X_{t}}^{X} \frac{\omega C_{0} \sigma}{C_{0}^{2}-U_{0}^{2}} \mathrm{~d} X^{\prime}\right)\right]
$$

where the first term represents the incident mode and the second the reflected mode. Beyond the transition point $X>X_{t}$, a cut-off attenuated mode is transmitted of the form

$$
\phi=N(X) \psi(r, \theta ; X) \exp \left(\frac{\mathrm{i}}{\varepsilon} \int_{X_{t}}^{X} \frac{\omega U_{0}}{C_{0}^{2}-U_{0}^{2}} \mathrm{~d} X^{\prime}\right) \exp \left(-\frac{1}{\varepsilon} \int_{X_{t}}^{X} \frac{\omega C_{0}|\sigma|}{C_{0}^{2}-U_{0}^{2}} \mathrm{~d} X^{\prime}\right),
$$

which does not propagate axially and carries no energy. It is important to remark here that the inclusion of the reflected mode is needed to conserve acoustic energy throughout the duct. ${ }^{6}$

The expressions Eq. (20) and Eq. (21) represent the so-called outer solution to the problem, as they are only valid away from the transition location $X_{t}$ when $\left|X-X_{t}\right| \sim 1$. In the neighbourhood $\left|X-X_{t}\right| \sim \varepsilon^{2 / 3}$ an inner solution holds to leading order, the non-convective axially varying part of which is a solution to Airy's equation. ${ }^{4,6}$ Whilst it is relatively straightforward to match the inner and outer solutions to obtain the required reflection coefficient, it is extremely difficult to use them alone to evaluate the resulting pressure and velocity perturbations inside the duct. This is because the inner region can take up a sizable proportional of the duct in reality and, of course, no exact axial station $X$ exists where the inner solution ceases to be valid and the outer solution can be substituted instead. Such a problem may be circumvented by finding a composite solution ${ }^{9}$ which is valid to leading order throughout the duct. For the case of an incident cut-on mode propagating in the positive $X$-direction described above, a composite multiple-scales solution for this mode can be derived to leading order of the form

$$
\phi=\bar{Q} \sqrt{\frac{C_{0}}{\omega D_{0}}} \psi(r, \theta ; X)\left[-\frac{3}{2 \varepsilon} \frac{1}{\sigma^{3}} \int_{X_{t}}^{X} \frac{\omega C_{0} \sigma}{C_{0}^{2}-U_{0}^{2}} \mathrm{~d} X^{\prime}\right]^{1 / 6} \mathrm{Ai}\left[\left(\frac{3 \mathrm{i}}{2 \varepsilon} \int_{X_{t}}^{X} \frac{\omega C_{0} \sigma}{C_{0}^{2}-U_{0}^{2}} \mathrm{~d} X^{\prime}\right)^{2 / 3}\right] \mathrm{e}^{\frac{\mathrm{i}}{\varepsilon} \int_{X_{t}}^{X} \frac{\omega U_{0}}{C_{0}^{2}-U_{0}^{2}} \mathrm{~d} X^{\prime}}
$$

Here, $\mathrm{Ai}$ is the Airy function of the first kind, and the eigensolution $\psi(r, \theta ; X)$ and the mean flow field are exactly as those determined for a normal slowly-varying mode in previous analyses. ${ }^{4,9}$ The constant $\bar{Q}$ is obtained from the source of the incident sound and differs from the $Q$ in Eq. (18) by some constants: $\bar{Q}=2 \sqrt{\pi} \mathrm{e}^{\frac{\mathrm{i} \pi}{4}} Q$. As one might expect, using the asymptotes of $\operatorname{Ai}(s)$ given in the appendix by Eqs. (28) it can be shown that in the limits $X \ll X_{t}$ and $X \gg X_{t}$, the composite solution, Eq. (22), tends to Eq. (20) and Eq. (21) respectively. 


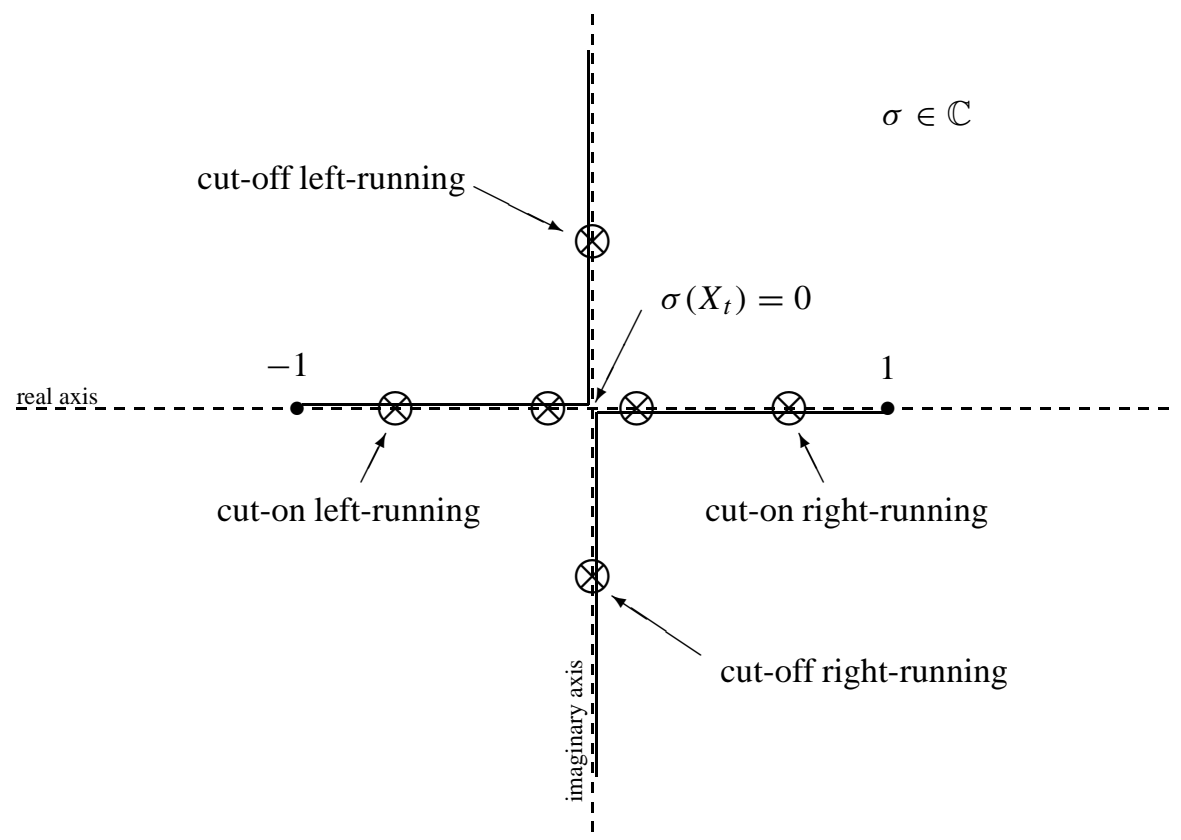

Figure ii. Sketch of the location (_ ${ }^{-}$of the reduced axial wavenumber of three right-running $(\sigma)$ and their corresponding left-running $(-\sigma)$ modes $(\otimes)$ in complex plane. The second mode is about the pass the turning point $X_{t}$, corresponding to $\sigma=0$.

\section{Cut-off cut-on transition}

A more general composite solution ${ }^{9}$ derived directly from the acoustic governing equation, Eq. (5), is

$$
\phi=\bar{Q} \sqrt{\frac{C_{0}}{\omega D_{0}}} \psi(r, \theta ; X)\left[-\frac{3}{2 \varepsilon} \frac{1}{\sigma^{3}} \int_{X_{t}}^{X} \frac{\omega C_{0} \sigma}{C_{0}^{2}-U_{0}^{2}} \mathrm{~d} X^{\prime}\right]^{1 / 6}\{a \operatorname{Ai}(s)+b \operatorname{Bi}(s)\} \mathrm{e}^{\frac{i}{\varepsilon} \int_{X_{t}}^{X} \frac{\omega U_{0}}{C_{0}^{2}-U_{0}^{2}} \mathrm{~d} X^{\prime}},
$$

for

$$
s=\left(\frac{3 \mathrm{i}}{2 \varepsilon} \int_{X_{t}}^{X} \frac{\omega C_{0} \sigma}{C_{0}^{2}-U_{0}^{2}} \mathrm{~d} X^{\prime}\right)^{2 / 3} .
$$

The arbitrary constants $a$ and $b$ are set by the two counterpart modes approaching $X_{t}$ from either side. From such a general solution we can derive cut-off cut-on transition, when an isolated cut-off mode propagating in the positive $X$-direction becomes cut-on at the point $X_{t}$. Clearly in this case, for $X>X_{t}$ and $\left|X-X_{t}\right| \sim 1$ we must only have a transmitted cut-on propagating mode ( $\sigma$ real and positive) of the form

$$
\phi=N(X) \psi(r, \theta ; X) \mathrm{e}^{\frac{\mathrm{i}}{\varepsilon} \int_{X_{t}}^{X} \frac{\omega U_{0}}{C_{0}^{2}-U_{0}^{2}} \mathrm{~d} X^{\prime}} \mathrm{e}^{-\frac{\mathrm{i}}{\varepsilon} \int_{X_{t}}^{X} \frac{\omega C_{0} \sigma}{C_{0}^{2}-U_{0}^{2}} \mathrm{~d} X^{\prime}} .
$$

From applying the large argument asymptotes in Eqs. (28) for both Airy functions $\operatorname{Ai}(s)$ and $\operatorname{Bi}(s)$ as $s \rightarrow-\infty$ (corresponding to $X>X_{t}$ and $\sigma$ real and positive), the required composite solution for cut-off cut-on transition satisfying Eq. (25) can be found and takes the form

$$
\phi=\sqrt{\pi} Q \sqrt{\frac{C_{0}}{\omega D_{0}}} \psi(r, \theta ; X)\left[-\frac{3}{2 \varepsilon} \frac{1}{\sigma^{3}} \int_{X_{t}}^{X} \frac{\omega C_{0} \sigma}{C_{0}^{2}-U_{0}^{2}} \mathrm{~d} X^{\prime}\right]^{1 / 6}\{\operatorname{Bi}(s)-\mathrm{i} \operatorname{Ai}(s)\} \mathrm{e}^{\frac{\mathrm{i}}{\varepsilon} \int_{X_{t}}^{X} \frac{\omega U_{0}}{C_{0}^{2}-U_{0}^{2}}} \mathrm{~d} X^{\prime} .
$$

\section{Finite-element solution}

The numerical model for duct propagation is based on a finite element (FEM) discretization of the steady flow field equations, Eqs. (4), and the acoustic field equations, Eq. (5) and Eq. (6) on the axisymmetric domain shown in Fig. iii. The computational domain consists of the defined duct geometry, shown between dashed boundaries, plus extensions which are required in the mean flow model to assure locally uniform flow. The acoustic source plane is at 


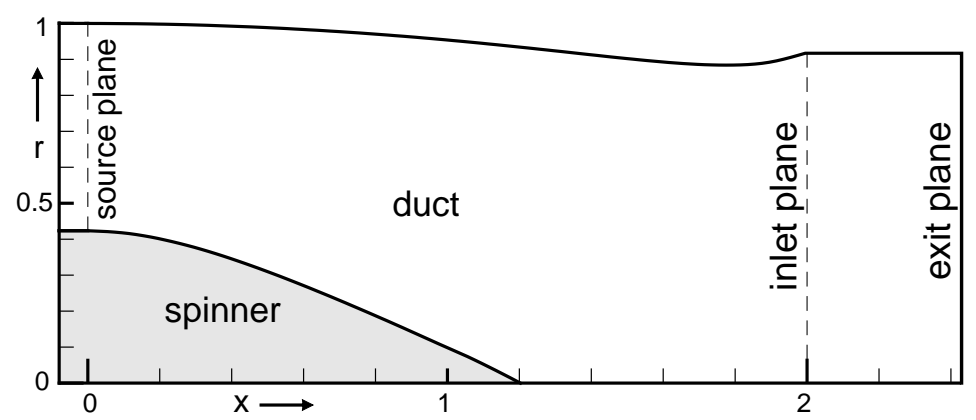

Figure iii. Calculational domain of FEM solution (including the lead-in)

the left domain boundary. The right domain boundary is extended from the nominal inlet plane, defined by the real duct geometry, to the exit plane where mean flow and acoustic boundary conditions are imposed.

\section{A. FEM model for duct propagation}

The steady compressible flow field is obtained from a Galerkin FEM formulation in terms of velocity potential of the first of equations Eqs. (4), the continuity equation, linearized at each step of an iterative process with density allowed to be spatially dependent. The second and third of equations Eqs. (4), the momentum equation and state equations, are subsidiary relations used to update the density and speed of sound at each step. Mass flow rate is specified on the source plane and the exit plane is assumed an equi-potential surface. The computational source and exit planes are generally placed a distance from the non-uniform region of the duct to assure locally uniform flow. In acoustic results presented here the source plane extension is 0.08 times the local outer duct radius, and at the termination plane the extension is 0.35 times the local duct radius. The mean flow field is described in terms of the mean flow velocity potential which is required as input data for the acoustic FEM model. The mean flow mesh is the same as the acoustic mesh to simplify data transfer.

The finite element model for acoustic propagation is also a Galerkin formulation based on the acoustic convected wave equation in terms of acoustic potential, Eq. (5). Equations (6) are the acoustic momentum (or energy) and state equations used to post process the acoustic potential to obtain the acoustic pressure and the acoustic density. The source is introduced at the source plane in terms of incident (right-running) acoustic potential modal amplitudes. Reflected (left-running) acoustic potential modal amplitudes are obtained as part of the solution. At the termination plane the acoustic field is represented by transmitted (right running) acoustic potential modal amplitudes and reflected (left running) acoustic potential modal amplitudes. The termination plane is assumed to be non-reflecting, and this is forced by requiring that reflected modal amplitudes vanish. Acoustic power is computed at the source plane and termination plane based on acoustic potential modal amplitudes by using the definition of Morfey ${ }^{13}$, valid in the case of irrotational acoustic perturbations on irrotational mean flow. In addition, acoustic power is computed at any specified axial location using the Morfey definition, but by post-processing the acoustic potential.

FEM modelling of acoustic propagation and radiation in non-uniform mean flow is presented in detail in previous works. ${ }^{10,14}$ More specific details of FEM applications to ducted flows terminated by reflection-free boundary conditions can be found elsewhere. ${ }^{15,16}$

\section{B. Comparison of the multiple scales (MS) and FEM formulations}

There are no differences in the field equations (3-6) used in the multiple scales solution and the finite element model, including the convention for non-dimensionalisation. The MS solution proceeds on the basis of the primitive variables, whereas FEM is in terms of mean flow and acoustic potentials, with acoustic pressure recovered by post-processing. In both formulations the source is introduced by acoustic modal amplitudes.

The FEM formulation admits scattering as an integral part of the solution, manifested at the source and termination planes by coupling between incident, transmitted and reflected modal amplitudes. In general there is observed reflection of the incident mode and other modes which are not incident as well as transmission of modes which are not incident. This will be clearly seen in examples which are presented. Such scattering is not such a direct feature of the multiple-scales solution. 
The ability of the MS solution to capture the essential details of the FEM solution is of principal interest in this investigation.

\section{Results}

The seven test cases considered examine a single radial acoustic mode undergoing transition from cut-on to cut-off (or vice-versa) at one axial location along the test geometry. The cases have realistic engine frequencies (Helmholtz numbers) $\omega$ lying in the range $11-66$ and realistic circumferential wavenumbers lying in the range $5-51$. Three of the cases are without mean flow to serve as useful benchmarks, whereas the other four have a mean flow which attains an axial mach number of 0.5 at the source plane. To ensure the mean flow profiles are equal for both methods at the source plane, the straight lead-in required for the FEM solution to the left of the test geometry is also included in the MS solution and its plots; see Figs. i and iii. For cases $1-6$, the mode in question undergoes cut-on cut-off transition and the finite-element (FEM) solution is compared to the multiple-scales (MS) composite solution given by Eq. (22). Case 7, however, is of an incident attenuated mode that cuts on close to the source plane, and here the FEM solution is compared to the appropriate composite solution given by Eq. (26).

For each test case, contour plots of absolute pressure are obtained from both FEM and MS solutions and plotted side-by-side for direct comparison. On the contour plot from the MS solution, a black dotted line is drawn at the axial location where transition is predicted to occur, i.e. at $x=x_{t}$. From the FEM solution, a data table is also provided for each case, containing the power and amplitudes (magnitude and phase) of all the relevant incident and reflected modes at the source plane as well as of all the transmitted modes at the termination plane. This data highlights the magnitude and extent of any modal scattering predicted by the FEM model.

Error estimates and the brief discussion later on the occurrence of modal scattering requires some estimate of the small parameter $\varepsilon$. This can be given by the typical slope of the duct geometry, which for the outer nacelle is about 0.1 . Of course, the slope of the inner spinner $\mathcal{O}\left(R_{1}^{\prime}(x)\right)$ is clearly larger than this. However, the spinner's centralness in the duct means that its slope is actually less important. This is due to the behaviour of the cross-sectional eigenfunction $\psi$ at small $r$, which goes like $r^{m}$ for circumferential order $m$. Therefore, taking $\varepsilon=0.1$ can be regarded as a highly reasonable estimate.

Case 1. No-flow with $m=21, \omega=41$ and $n=4$

We start with a benchmark case (Fig. 1) without mean flow, but with realistic $m$ and $\omega$ in aero-engine applications. Transition point occurs (from MS) at $x_{t}=1.49$. The agreement is evidently excellent. The WKB assumption of no exchange of energy between other radial modes than the incident and its mirror reflection is confirmed by Table 1 of modal powers (from FEM). Note important features such as (i) interference of incident and reflected modes creates the bumps in absolute pressure implying a standing wave ${ }^{8}$; (ii) the largest pressure rise occurs just ahead of transition due to Airy function's behaviour.

Case 2. $M=0.5$ with $m=10, \omega=11$ and $n=1$

The second case is with low radial mode $n=1$, relatively low $m$ and $\omega$, but with strong mean flow (Fig. 2). It is based on a case already attempted by Thiele et al. ${ }^{17}$, which provides a further comparison. The inner solution appears to span the majority (if not all) of the duct. From Table 2 we see that no scattering into neighbouring modes occurs. The unadjusted MS solution $(M=0.5$ and $\omega=11)$ is already very similar to the FEM solution, but is slightly receded. We speculate (given the excellent agreement of the no-flow case above and the typical error in the approximate MS mean flow of $\mathcal{O}\left(\varepsilon^{2}\right)$ which is here a few percent) that this may largely be explained by the slight mean flow differences between MS and FEM. The MS solution can be 'tuned' to achieve a better match, either by adjusting the frequency or the mean flow. The position of the turning point is highly sensitive; $2-3 \%$ mean flow and $<1 \%$ frequency alteration is required. Indeed, Thiele et al. also found for a similar configuration $(\omega=11.129)$ the same features including a highly sensitive position of the turning point to mean flow variations.

This is easily explained by noting that the position of $X_{t}$ is determined by $\sigma\left(X_{t}\right)=0$ with Eqs. (9), (16) and (19), which may be considered as a set of algebraic equations in $M, \omega$ and $X$. This means that

$$
X_{t}(M+\Delta M, \omega+\Delta \omega)=X_{t}(M, \omega)+\mathcal{O}(\Delta M)+\mathcal{O}(\Delta \omega) .
$$

If $\Delta M=\mathcal{O}\left(\varepsilon^{2}\right)$ (which may be expected), the error in $X_{t}$ is also $\mathcal{O}\left(\varepsilon^{2}\right)$, and thus the error in $x_{t}$ is $\mathcal{O}(\varepsilon)$, in other words, in the order of $10 \%$, which is indeed what we observe. In the same way it is clear that only a shift of $\mathcal{O}\left(\varepsilon^{2}\right)$ in either $M$ or $\omega$ should suffice for readjustment. 
Case 3. No-flow with $m=51, \omega=66$ and $n=2$

The next case is for no flow but with high $m$ and $\omega$; see Fig. 3. Excellent agreement once again is obtained in terms of absolute pressure and prediction of the transition point location and modal reflection, while Table 3 confirms absence of modal scattering.

Case 4. $M=0.5$ with $m=51, \omega=57.5$ and $n=2$

The fourth case, Fig. 4, is relatively difficult numerically, with realistic high frequency and mean flow. The agreement is again very good despite the transition point in the MS solution being slightly to the left of that in the FEM solution. Table 4 shows no significant modal scattering.

Case 5. No-flow with $m=20, \omega=50.2$ and $n=7$

This case, Fig. 5, examines the composite solution's behaviour with high radial order modes. For this no-flow case there is very little if no scattering perceivable (only about $1 \%$ of the power leaks into mode 6 ; see Table 5) and the agreement is excellent. However, compare this to the a high-radial order case with mean flow below (case 6).

Case 6. $M=0.5$ with $m=20, \omega=44.4$ and $n=7$

This case, Fig. 6, is for a mode of high radial order and high frequency and large mean flow. Prediction of the transition point seems to be fairly accurate but notice (Table 6) the large amount of scattering into the neighbouring cut-on modes ( $n=6$-transmitted, $n=8$-reflected).

Case 7. $M=0.5$ with $m=5, \omega=19.8$ and $n=5$

Finally, in Fig. 7, we present a challenging cut-off, cut-on transition case obtained from the other composite solution given by Eq. (26). An incident cut-off mode rapidly cuts-on a short distance away from the source plane $\left(x_{t}=0.18\right)$ and a reflected cut-off mode must be produced. The two solutions show similar features, such as large pressure amplitudes over the surface of the spinner and in the hollow part close to the centre line. Some scatter is apparent into other radial modes; see Table 7.

\section{Some thoughts on modal scattering}

Suppose we have two slowly varying modes, which are independent (albeit approximate) solutions of the problem whenever they are non-singular.

$$
\begin{array}{r}
\phi=\left\{\frac{A_{1}}{\sqrt{\sigma_{1}(X)}} \psi_{1}(r, \theta ; X) \exp \left(-\frac{\mathrm{i}}{\varepsilon} \int_{X_{t}}^{X} \frac{\omega C_{0} \sigma_{1}}{C_{0}^{2}-U_{0}^{2}} \mathrm{~d} X^{\prime}\right)+\frac{A_{2}}{\sqrt{\sigma_{2}(X)}} \psi_{2}(r, \theta ; X) \exp \left(-\frac{\mathrm{i}}{\varepsilon} \int_{X_{t}}^{X} \frac{\omega C_{0} \sigma_{2}}{C_{0}^{2}-U_{0}^{2}} \mathrm{~d} X^{\prime}\right)\right\} \ldots \\
\cdot\left(\frac{C_{0}}{\omega D_{0}}\right)^{1 / 2} \exp \left(\frac{\mathrm{i}}{\varepsilon} \int_{X_{t}}^{X} \frac{\omega U_{0}}{C_{0}^{2}-U_{0}^{2}} \mathrm{~d} X^{\prime}\right) .
\end{array}
$$

From the linearity of the problem it follows that no interaction with each other is necessary or to be expected. However, this changes near a turning point where (say) $\sigma_{1}=0$ and mode 1 has to share its energy with other modes because (a) it is not any more an approximate solution of the problem and (b) the energy flux associated with mode 1 vanishes beyond the turning point.

In order for mode 2 to exchange energy with mode 1 , it has to be as singular, or at least of the same order of magnitude, as mode 1 . The obvious first candidate is the mirror (the same but opposite-running) mode, of which $\sigma_{2}=\sigma_{1}=0$. This is however, not always the only possible candidate.

So we are posed the question: suppose that mode 1 and mode 2 are neighbouring modes, under what conditions does $\sigma_{2} \sim \sigma_{1}$ in the turning point boundary layer (given that order of magnitude estimates of all other factors do not alter inside the boundary layer)?

First we note from the definition, Eq. (19), of $\sigma$ that for $\sigma_{1}=0$ and $\sigma_{2}$ to be nearly zero, both $\alpha_{1}$ and $\alpha_{2}$ must be approximately equal to $\left(C_{0}^{2}-U_{0}^{2}\right)^{1 / 2} \omega$, which is only possible (if the duct diameter is not very small) if both $n$ and $\omega$ are large; this is certainly true in our examples demonstrating modal scattering. 
From the previous analyses ${ }^{4,6,9}$ it follows that in the boundary layer,

$$
X=X_{t}+\varepsilon^{2 / 3} \lambda^{-1} \xi
$$

we have

$$
\sigma_{1}^{2}(X)=\sigma_{1}^{2}\left(X_{t}+\varepsilon^{2 / 3} \lambda^{-1} \xi\right)=-2 \varepsilon^{2 / 3}\left(\frac{C_{0 t} C_{0 t}^{\prime}-U_{0 t} U_{0 t}^{\prime}}{C_{0 t}^{2}-U_{0 t}^{2}}+\frac{\alpha_{t}^{\prime}}{\alpha_{t}}\right) \lambda^{-1} \xi+\mathcal{O}\left(\varepsilon^{4 / 3} \xi^{2}\right),
$$

where subscript ${ }_{t}$ indicates evaluation at $X=X_{t}$ and $\lambda$ is given by

$$
\lambda^{3}=\frac{2 \omega^{2} C_{0 t}^{2}}{\left(C_{0 t}^{2}-U_{0 t}^{2}\right)^{2}}\left(\frac{C_{0 t} C_{0 t}^{\prime}-U_{0 t} U_{0 t}^{\prime}}{C_{0 t}^{2}-U_{0 t}^{2}}+\frac{\alpha_{t}^{\prime}}{\alpha_{t}}\right) .
$$

In the original analysis $\lambda=\mathcal{O}(1)$ by assumption, but this is not fully satisfied here as $\omega$ is large. For the question: when is $\sigma_{2} \sim \sigma_{1}$, we first note that

$$
\frac{1-\sigma_{1}^{2}}{\alpha_{1}^{2}}=\frac{1-\sigma_{2}^{2}}{\alpha_{2}^{2}}
$$

from which we immediately get

$$
\sigma_{2}^{2}=\frac{\alpha_{1}^{2}-\alpha_{2}^{2}}{\alpha_{1}^{2}}+\frac{\alpha_{2}^{2}}{\alpha_{1}^{2}} \sigma_{1}^{2} .
$$

For large order, the difference $\Delta \alpha$ between consecutive eigenvalues tends to a constant, so $\sigma_{2}$ is of the same order as $\sigma_{1}$ if:

$$
\frac{\Delta \alpha}{\alpha} \simeq \frac{1}{2} \sigma_{1}^{2}=\varepsilon^{2 / 3} \lambda^{2} \frac{\left(C_{0}^{2}-U_{0}^{2}\right)^{2}}{2 \omega^{2} C_{0}^{2}} \xi
$$

Since normally the term $\alpha^{\prime} / \alpha$ in $\lambda$ dominates, we have

$$
\frac{\Delta \alpha}{\alpha} \simeq \varepsilon^{2 / 3}\left(\frac{\alpha^{\prime}}{\alpha}\right)^{2 / 3} \frac{\left(C_{0}^{2}-U_{0}^{2}\right)^{2 / 3}}{\left(2 \omega^{2} C_{0}^{2}\right)^{1 / 3}} \xi .
$$

As $\omega \simeq\left(C_{0}^{2}-U_{0}^{2}\right)^{1 / 2} \alpha$, this turns into

$$
\omega \simeq \varepsilon^{-2} \frac{2(\Delta \alpha)^{3} C_{0}^{2}}{\left(\alpha^{\prime} / \alpha\right)^{2}\left(C_{0}^{2}-U_{0}^{2}\right)^{1 / 2} \xi^{3}} .
$$

For a hollow duct we have for large $\operatorname{order}^{18} \alpha=j_{m n}^{\prime} / R(X) \simeq\left(n+\frac{1}{2} m-\frac{3}{4}\right) \pi / R$ and so $\Delta \alpha=\pi / R$. For an annular duct of hub-tip ratio $h$ we have $\alpha \simeq n \pi /(1-h) R$ and so $\Delta \alpha=\pi /(1-h) R$. In either case we see that for $n$ and $\omega$ high enough indeed modal scattering may be expected. The order of magnitude $\omega=\mathcal{O}\left(\varepsilon^{-2}\right)$ is indeed consistent with our $\varepsilon=0.1$ and $\omega \sim 50$ of the cases in question where scattering was observed.

\section{Conclusions}

A theoretical framework has been established for the modelling of the propagation of sound in a non-uniform duct with mean subsonic compressible flow in the case when the incident mode of propagation encounters a cut-off or cuton transition. The analysis is based on the assumption that the duct geometry and flow field are slowly varying, leading to application of the method of multiple scales. Far away from the transition the multiple scales solution consists of slowly varying duct modes. At the transition they become singular, and a local solution is necessary to connect the cut-on and the cut-off parts of the mode. This set of local solutions is impractical, but it has been shown possible to construct a composite solution, encompassing the boundary layer solution in the neighbourhood of the transition point and the outer slowly varying modal solution far from the transition into a single expression. Such a theoretical model has been benchmarked here by comparison with a fully numerical model based on the finite element method.

Cases used for our comparison are varied, with and without mean flow, and over a range of circumferential modes, radial modes and frequencies. Cases include those with as few as one incident propagating mode to as many as eight propagating modes, and include a particularly interesting case with an incident non-propagating mode (cut-off at the source) which cuts on close to the source. 
Comparisons between multiple scales results and finite element results are presented in the form of contours of equal pressure magnitude, which for most cases show the standing wave character associated with the analytically predicted complete reflection of the incident mode into its mirror image propagating in the opposite direction. Contour plots are supplemented with tables of incident, reflected, and transmitted modal power and reflected and transmitted modal amplitudes generated by the finite element model. The finite element solution always shows some scattering into reflected and transmitted modes adjacent to the incident modes. This scattering is small in most instances, in accordance with the assumptions of the multiple scales analysis. In these cases the agreement between FEM and the multiple scales solution is invariably good (with flow) or excellent (without flow). An explanation for this difference, which is consistent with theory, is that the accuracy of the analytical mean flow $\left(\mathcal{O}\left(\varepsilon^{2}\right)\right.$, typically a few percent) is seen to produce an error of $\mathcal{O}(\varepsilon)$, of the order of 10 percent, in the position of the transition.

In some cases, one of which is shown here, the finite element solution shows significant scattering into adjacent modes, which is not predicted by the multiple scales solution. In our study such an occurrence was limited to cases with mean flow and with many initially propagating modes (eight in the case shown here), with the highest order modes having cut-off ratios which cluster near unity. In these cases with scattering, the multiple scales solution captures the basic features of the acoustic field, but does not predict the scattering mechanism by which acoustic power leaks through the turning point.

The observation of significant scattering in certain cases has led to an extension of the multiple scales analysis by asymptotic scaling arguments in the turning point region. This has identified circumstances under which the presence of neighbouring modes is necessary and modal interaction is likely to occur.

The challenging case of an incident mode, cut-off at the source, and cutting on close to the source, shows generally good agreement between the multiple-scales solution and the finite-element solution, with FEM showing evidence of scattering into adjacent modes. This, however, does not seem to be a particular property of this configuration.

Aside from the benchmark comparisons which were the main thrust of the current investigation, calculations of the standing wave field associated with cut-off phenomenon revealed high acoustic pressures in the neighbourhood of the turning point. This may well have implications for nacelle structural integrity and structure-borne noise.

\section{Appendix}

Related to Bessel functions of order $\frac{1}{3}$ are the Airy functions ${ }^{18} \mathrm{Ai}$ and $\mathrm{Bi}$, solutions of $y^{\prime \prime}-x y=0$, with the following asymptotic behaviour (introduce $\zeta=\frac{2}{3}|x|^{3 / 2}$ )

$$
\begin{aligned}
& \operatorname{Ai}(x) \simeq \frac{\cos \left(\zeta-\frac{1}{4} \pi\right)}{\sqrt{\pi}|x|^{1 / 4}} \quad(x \rightarrow-\infty), \quad \simeq \frac{\mathrm{e}^{-\zeta}}{2 \sqrt{\pi} x^{1 / 4}} \quad(x \rightarrow \infty), \\
& \operatorname{Bi}(x) \simeq \frac{\cos \left(\zeta+\frac{1}{4} \pi\right)}{\sqrt{\pi}|x|^{1 / 4}} \quad(x \rightarrow-\infty), \quad \simeq \frac{\mathrm{e}^{\zeta}}{\sqrt{\pi} x^{1 / 4}} \quad(x \rightarrow \infty) .
\end{aligned}
$$

\section{Acknowledgements}

N.C. Ovenden would like to thank UCL Graduate School, the Department of Mathematics at UCL and Eindhoven University of Technology for financial support.

S.W. Rienstra's contribution was partly carried out in the context of the "Messiaen" project of the European Union's 6th Framework and partly under a grant of the Royal Society at the University of Cambridge. The financial support of both parties is greatly acknowledged.

We wish to thank Nigel Peake (University of Cambridge) for his stimulating interest and useful suggestions and remarks.

Finally, we wish to thank Frank Thiele and his group at the Technical University of Berlin for their initiating the problem along with their enthusiasm and interest.

\section{References}

\footnotetext{
${ }^{1}$ A.H. Nayfeh and D.P. Telionis, "Acoustic propagation in ducts of varying cross sections." Journal of the Acoustical Society of America $\mathbf{5 4}$ (1973) 1654-1661.

${ }^{2}$ S.W. Rienstra, "Sound transmission in slowly varying circular and annular lined ducts with flow." Journal of Fluid Mechanics 380 (1999) 279-296.
} 
${ }^{3}$ A.J. Cooper and N. Peake, "Propagation of unsteady disturbances in a slowly varying duct with mean swirling flow." Journal of Fluid Mechanics 445 (2001) 207-234.

${ }^{4}$ S.W. Rienstra, "Sound propagation in slowly varying lined flow ducts of arbitrary cross section." Journal of Fluid Mechanics 495 (2003), $157-173$

${ }^{5}$ S.W. Rienstra and W. Eversman, "A numerical comparison between the multiple-scales and finite-element solution for sound propagation in lined flow ducts." Journal of Fluid Mechanics 437 (2001) 367-384.

${ }^{6}$ S.W. Rienstra, "Cut-on cut-off transition of sound in slowly varying flow ducts." Aerotechnica - Missili e Spazio, special issue in memory of David Crighton. (edited by L. Morino and N. Peake) 79, nos. 3-4, (2000) 93-97.

${ }^{7}$ N.C. Ovenden, "Near cut-on/cut-off transitions in lined ducts with flow." Paper AIAA 2002-2445 of the 8th AIAA/CEAS Aeroacoustics Conference in Breckenridge, CO, 17-19 June (2002).

${ }^{8}$ A.J. Cooper and N. Peake, "Trapped acoustic modes in aeroengine intakes with swirling flow." Journal of Fluid Mechanics 419 (2000) $151-175$.

${ }^{9}$ N.C. Ovenden, "A composite multiple-scales solution for cut-on cut-off transition in a hard-walled duct with flow." submitted to Journal of Sound and Vibration (2004).

${ }^{10}$ I. Danda Roy and W. Eversman, "Improved finite element modeling of the turbofan engine inlet radiation problem." ASME Journal of Vibration and Acoustics 117 (1995), 109-115.

${ }^{11}$ M. Van Dyke, "Slow Variations in Continuum Mechanics", in: Advances in Applied Mechanics 25, 1-45, Academic Press, Orlando (1987)

${ }^{12}$ A.H. Nayfeh, Perturbation Methods. John Wiley Sons Inc., New York (1973)

${ }^{13}$ C.L. Morfey, "Acoustic energy in non-uniform flows." Journal of Sound and Vibration 14, (1971), 159-170.

${ }^{14}$ I. Danda Roy and W. Eversman, "Far field calculations for turbofan noise." AIAA Journal 39(12), (2001), 2255-2261.

${ }^{15}$ W. Eversman, "A reverse flow theorem and acoustic reciprocity in compressible potential flow in ducts." Journal of Sound and Vibration 246(1), (2001), 71-95.

${ }^{16}$ W. Eversman, "Numerical experiments on acoustic reciprocity in compressible potential flows in ducts." Journal of Sound and Vibration 246(1), (2001), 97-113.

${ }^{17}$ X.D. Li, C. Schemel, U. Michel and F. Thiele, "On the azimuthal mode propagation in axisymmetric duct flows." Paper $2002-2521$ of the 8th AIAA/CEAS Aeroacoustics Conference in Breckenridge, CO, USA, 17-19 June (2002). In revised form, entitled: "On the azimuthal sound mode propagation in axisymmetric flow ducts", accepted for publication in AIAA-Journal (2004).

${ }^{18}$ M. Abramowitz and I.A. Stegun, Handbook of Mathematical Functions, National Bureau of Standards, Dover Publications, Inc., New York (1964) 




(a) FEM

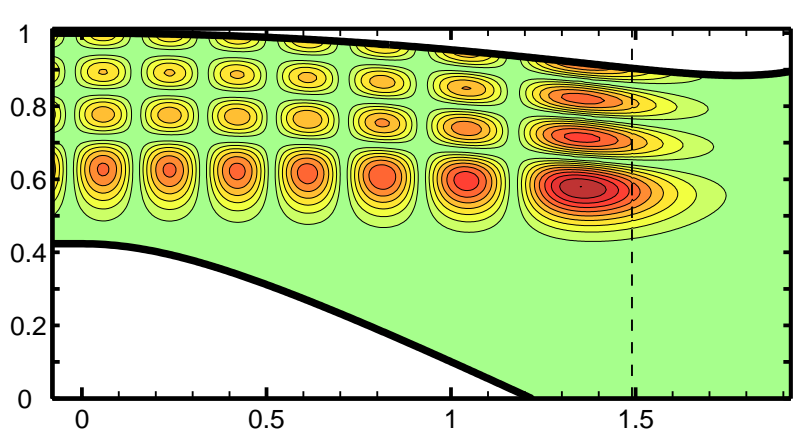

(b) MS

Figure 1. No-flow with $m=21, \omega=41.0$ and $n=4$. Transition at $x_{t}=1.49$.

Table 1. FEM modal amplitudes and modal power for $M=0.0, m=21, \omega=41.0, n=4$

modal amplitudes (modulus and phase)

modal powers

\begin{tabular}{|c|c|c|c|c|c|c|c|c|}
\hline$n$ & input & \multicolumn{2}{|c|}{ reflected } & \multicolumn{2}{|c|}{ transmitted* } & input & reflected & transmitted \\
\hline 1 & 0.00 & $1.28806^{-03}$ & -0.16011 & $4.65308^{-03}$ & -1.00415 & 0.0000 & $1.2609-07$ & $1.3199-06$ \\
\hline 2 & 0.00 & $1.24518^{-03}$ & -2.07156 & $7.59902-03$ & -2.41687 & 0.0000 & $1.4446^{-07}$ & $4.1023-06$ \\
\hline 3 & 0.00 & $1.49994^{-02}$ & 1.32924 & $1.78118^{-02}$ & -2.74923 & 0.0000 & $1.7160-05$ & $1.6330-05$ \\
\hline 4 & 1.00 & $9.98636-01$ & 1.73083 & $7.33522-02$ & 0.73351 & $5.2870-02$ & $5.2734-02$ & $9.4760-05$ \\
\hline 5 & 0.00 & $1.30240^{-02}$ & -1.43072 & $1.96820-05$ & -2.72645 & 0.0000 & $2.1353-06$ & 0.0000 \\
\hline 6 & 0.00 & $7.97316^{-05}$ & 0.84398 & $2.66586^{-07}$ & 0.20589 & 0.0000 & 0.0000 & 0.0000 \\
\hline 7 & 0.00 & $1.49995-05$ & 1.13319 & $1.25423-08$ & -3.04410 & 0.0000 & 0.0000 & 0.0000 \\
\hline
\end{tabular}

* Phase of transmitted mode is relative to exit plane.

Table 2. FEM modal amplitudes and modal power for $M=0.5, m=10, \omega=11.0, n=1$

modal amplitudes (modulus and phase)

modal powers

\begin{tabular}{c|c|rr|rr|l|l|c}
\hline$n$ & \multicolumn{2}{|c}{ input } & \multicolumn{2}{|c|}{ reflected } & \multicolumn{2}{c}{ transmitted $^{*}$} & \multicolumn{1}{c}{ input } & \multicolumn{2}{c}{ reflected } & transmitted \\
\hline 1 & 1.00 & $6.83653-01$ & 0.31240 & $4.95448-02$ & -1.34561 & $2.0844-02$ & $2.0844-02$ & 0.0000 \\
2 & 0.00 & $8.13841-03$ & -0.67365 & $1.58488-04$ & -2.14545 & 0.0000 & 0.0000 & 0.0000 \\
3 & 0.00 & $6.58219-04$ & 2.66479 & $1.49392-05$ & 0.74636 & 0.0000 & 0.0000 & 0.0000
\end{tabular}

* Phase of transmitted mode is relative to exit plane. 


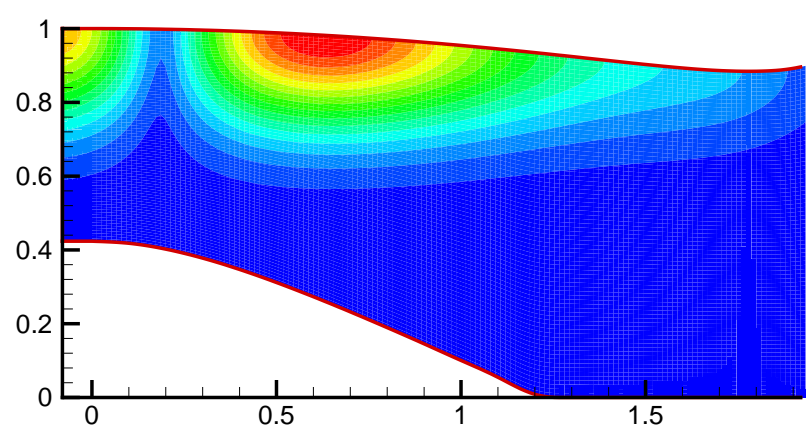

(a) FEM, $M=0.5, \omega=11.0$

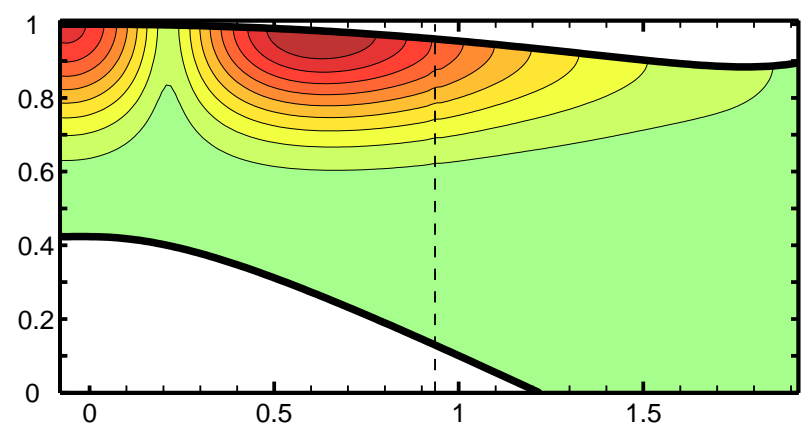

(c) MS, $M=0.5, \omega=11.08$, transition at $x_{t}=0.94$.

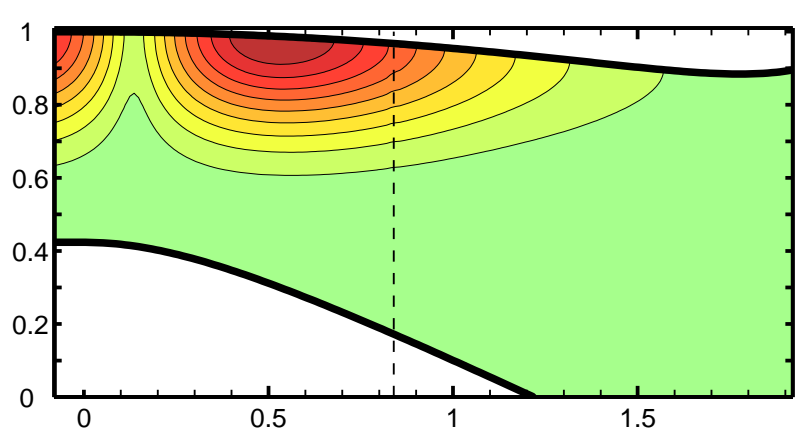

(b) MS, $M=0.5, \omega=11.0$, transition at $x_{t}=0.84$.

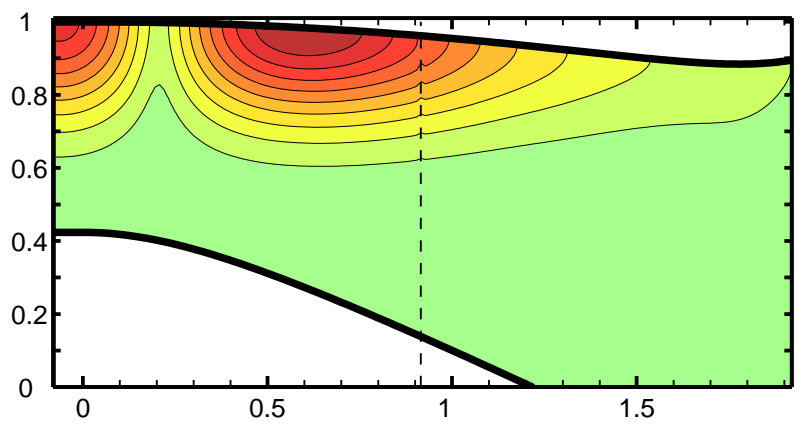

(d) MS, $M=0.514, \omega=11.0$, transition at $x_{t}=0.92$.

Figure 2. $M=0.5$ with $m=10, \omega=11.0$ and $n=1$. Transition at $x_{t}=0.84$ (unadjusted), at $x_{t}=0.94\left(\omega\right.$ adjusted) and at $x_{t}=0.92(M$ adjusted).

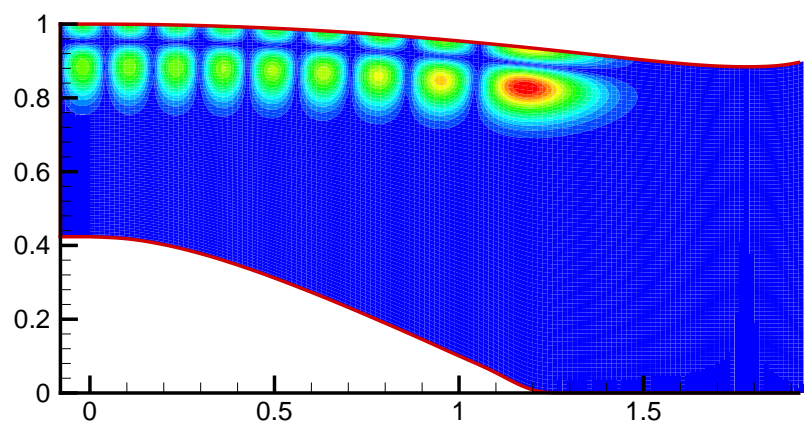

(a) FEM



(b) MS

Figure 3. No-flow with $m=51, \omega=66.0$ and $n=2$. Transition at $x_{t}=1.29$. 
Table 3. FEM modal amplitudes and modal power for $M=0.0, m=51, \omega=66.0, n=2$

modal amplitudes (modulus and phase)

modal powers

\begin{tabular}{c|c|rr|rr|l|l|l}
\hline$n$ & \multicolumn{7}{|c}{ input } & \multicolumn{2}{c|}{ reflected } & \multicolumn{2}{c|}{ transmitted } & \multicolumn{1}{c}{ input } & \multicolumn{1}{c}{ reflected } & \multicolumn{1}{c}{ transmitted } \\
\hline 1 & 0.00 & $6.72025-03$ & 2.25428 & $7.97928-03$ & 0.58151 & 0.0000 & $1.4091-06$ & $1.3114-06$ \\
2 & 1.00 & $9.99891-01$ & -3.03406 & $7.10642-05$ & 0.79078 & $3.3347-02$ & $3.3344-02$ & 0.0000 \\
3 & 0.00 & $9.32808-04$ & 1.64194 & $6.79870-09$ & 3.04031 & 0.0000 & 0.0000 & 0.0000 \\
4 & 0.00 & $8.70045-05$ & -1.51414 & $3.34634-09$ & -1.99459 & 0.0000 & 0.0000 & 0.0000
\end{tabular}

* Phase of transmitted mode is relative to exit plane.

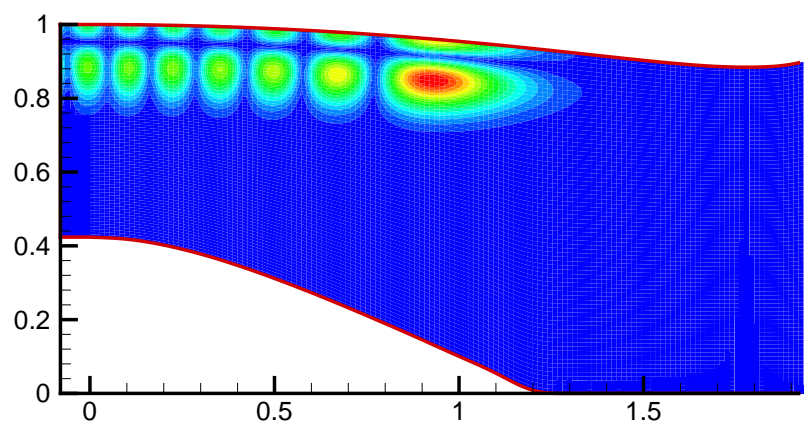

(a) FEM

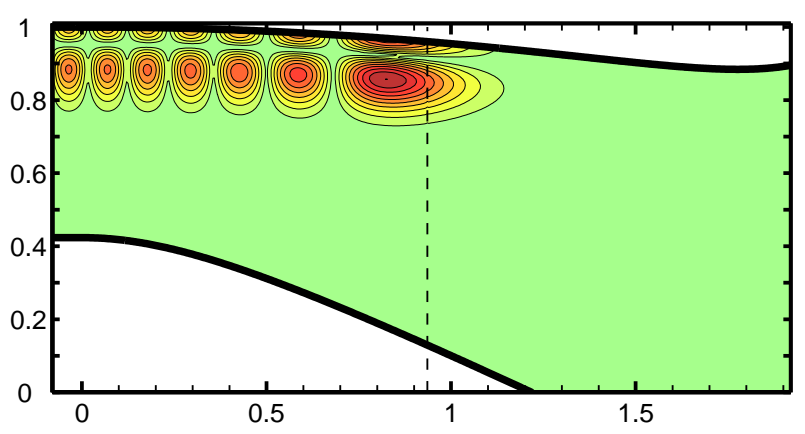

(b) MS

Figure 4. $M=0.5$ with $m=51, \omega=57.5$ and $n=2$. Transition at $x_{t}=0.9356$ (unadjusted).

Table 4. FEM modal amplitudes and modal power for $M=0.5, m=51, \omega=57.5, n=2$

modal amplitudes (modulus and phase)

modal powers

\begin{tabular}{c|c|cc|cc|c|c|c}
\hline$n$ & \multicolumn{2}{|c}{ input } & \multicolumn{2}{c|}{ reflected } & \multicolumn{2}{c|}{ transmitted } & \multicolumn{1}{c}{ input } & \multicolumn{2}{c}{ reflected } & transmitted \\
\hline 1 & 0.00 & $4.64375-03$ & 0.25482 & $2.33365-02$ & -2.18438 & 0.0000 & $7.6128-07$ & $4.3142-06$ \\
2 & 1.00 & $6.71150-01$ & 1.91208 & $7.01745-05$ & -0.88275 & $1.3573-02$ & $1.3525-02$ & 0.0000 \\
3 & 0.00 & $1.54454-01$ & 2.41438 & $1.53737-06$ & 0.93476 & 0.0000 & $4.3465-05$ & 0.0000 \\
4 & 0.00 & $3.86885-03$ & 1.23189 & $2.86459-07$ & -2.59817 & 0.0000 & 0.0000 & 0.0000 \\
5 & 0.00 & $2.47703-04$ & -1.30422 & $1.03903-07$ & -0.08191 & 0.0000 & 0.0000 & 0.0000
\end{tabular}

* Phase of transmitted mode is relative to exit plane. 


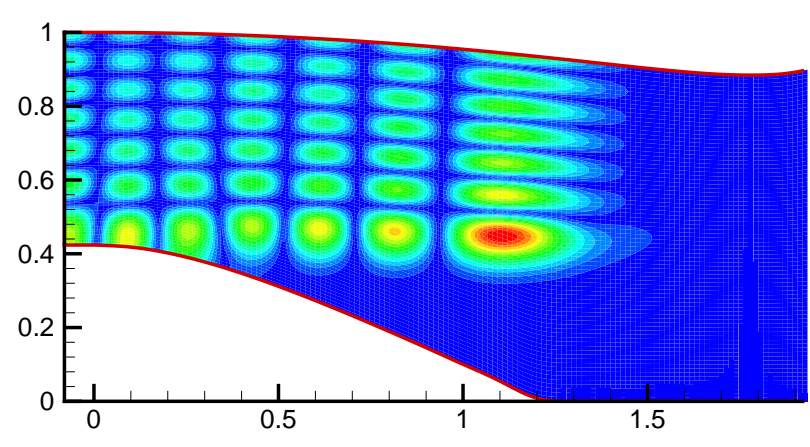

(a) FEM

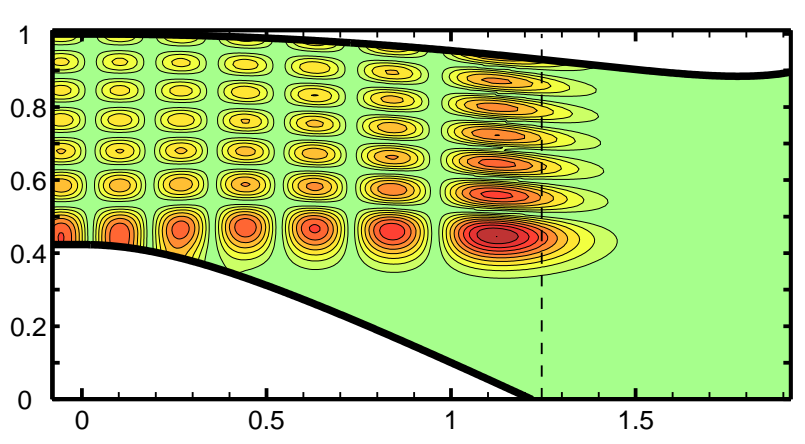

(b) MS

Figure 5. No-flow with $m=20, \omega=50.2$ and $n=7$. Transition at $x_{t}=1.25$.

Table 5. FEM modal amplitudes and modal power for $M=0.0, m=20, \omega=50.2, n=7$

modal amplitudes (modulus and phase)

modal powers

\begin{tabular}{|c|c|c|c|c|c|c|c|c|}
\hline$n$ & input & \multicolumn{2}{|c|}{ reflected } & \multicolumn{2}{|c|}{ transmitted* } & input & reflected & transmitted \\
\hline 1 & 0.00 & $9.79832-04$ & 1.75305 & $5.67365-03$ & -1.81000 & 0.0000 & $8.1703-08$ & $2.2494-06$ \\
\hline 2 & 0.00 & $6.20071-04$ & -0.19682 & $4.71254-03$ & 2.91263 & 0.0000 & $4.2842-08$ & $1.9896-06$ \\
\hline 3 & 0.00 & $5.73870^{-04}$ & -2.00684 & $6.47028-03$ & 1.44566 & 0.0000 & $3.3281-08$ & $3.3317-06$ \\
\hline 4 & 0.00 & $1.95037-03$ & -2.97036 & $1.00937-02$ & 0.31196 & 0.0000 & $3.3145-07$ & $6.7235-06$ \\
\hline 5 & 0.00 & $9.79360-03$ & 2.84038 & $1.85307-02$ & -0.49332 & 0.0000 & $7.0020-06$ & $1.7260-05$ \\
\hline 6 & 0.00 & $7.47880-02$ & -1.97637 & $9.14736-02$ & 0.18077 & 0.0000 & $3.1977-04$ & $2.6909-04$ \\
\hline 7 & 1.00 & $9.85999-01$ & -0.49319 & $1.57594-03$ & 1.33494 & $3.3261-02$ & $3.2341-02$ & 0.0000 \\
\hline 8 & 0.00 & $1.71606^{-01}$ & 2.38235 & $2.57213-06$ & -2.40985 & 0.0000 & $2.9241-04$ & 0.0000 \\
\hline 9 & 0.00 & $5.70550^{-04}$ & -3.00552 & $7.25713-08$ & 0.53864 & 0.0000 & 0.0000 & 0.0000 \\
\hline 10 & 0.00 & $2.76103-04$ & 2.95400 & $6.76850-09$ & 0.72034 & 0.0000 & 0.0000 & 0.0000 \\
\hline
\end{tabular}

* Phase of transmitted mode is relative to exit plane.

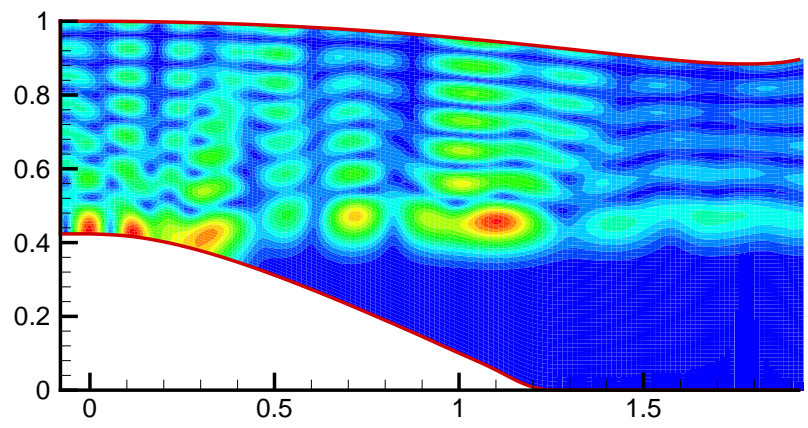

(a) FEM

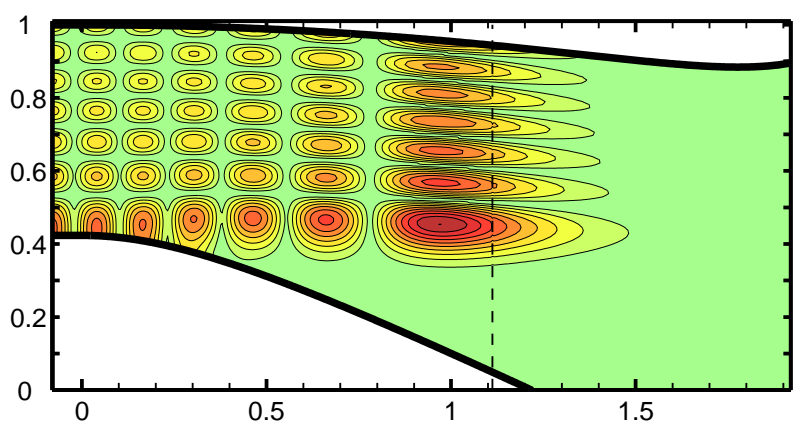

(b) MS

Figure 6. $M=0.5$ with $m=20, \omega=44.4$ and $n=7$. Transition at $x_{t}=1.11$. 
Table 6. FEM modal amplitudes and modal power for $M=0.5, m=20, \omega=44.4, n=7$

modal amplitudes (modulus and phase)

modal powers

\begin{tabular}{|c|c|c|c|c|c|c|c|c|}
\hline$n$ & input & \multicolumn{2}{|c|}{ reflected } & \multicolumn{2}{|c|}{ transmitted* } & input & reflected & transmitted \\
\hline 1 & 0.00 & $2.60377-03$ & -2.49574 & $1.05164-02$ & 2.05856 & 0.0000 & $1.0804-06$ & $2.1964-06$ \\
\hline 2 & 0.00 & $1.93046^{-03}$ & 2.67222 & $7.54334-03$ & 0.75997 & 0.0000 & $7.0209-07$ & $1.5307-06$ \\
\hline 3 & 0.00 & $2.53907-03$ & 1.64990 & $8.62162-03$ & 0.35758 & 0.0000 & $1.0013^{-06}$ & $1.8830-06$ \\
\hline 4 & 0.00 & $4.05321-03$ & 2.28672 & $4.92354-02$ & 1.38552 & 0.0000 & $1.9903-06$ & $5.4733-05$ \\
\hline 5 & 0.00 & $1.51628-02$ & -1.68721 & $9.40746-02$ & -2.59292 & 0.0000 & $2.0986-05$ & $1.6789-04$ \\
\hline 6 & 0.00 & $6.02846^{-02}$ & -0.33907 & $4.83654-01$ & -2.98763 & 0.0000 & $2.3334-04$ & $3.3562-03$ \\
\hline 7 & 1.00 & $3.34318^{-01}$ & 2.46029 & $3.65047-02$ & -1.37429 & $1.3976^{-02}$ & $3.7818^{-03}$ & 0.0000 \\
\hline 8 & 0.00 & $6.99441-01$ & 2.60611 & $1.04325-03$ & -0.35725 & 0.0000 & $6.3504-03$ & 0.0000 \\
\hline 9 & 0.00 & $3.28409-02$ & 2.01446 & $9.62889-05$ & 2.55026 & 0.0000 & 0.0000 & 0.0000 \\
\hline 10 & 0.00 & $3.96434-04$ & 0.65910 & $1.16562-05$ & -0.75301 & 0.0000 & 0.0000 & 0.0000 \\
\hline
\end{tabular}

* Phase of transmitted mode is relative to exit plane.

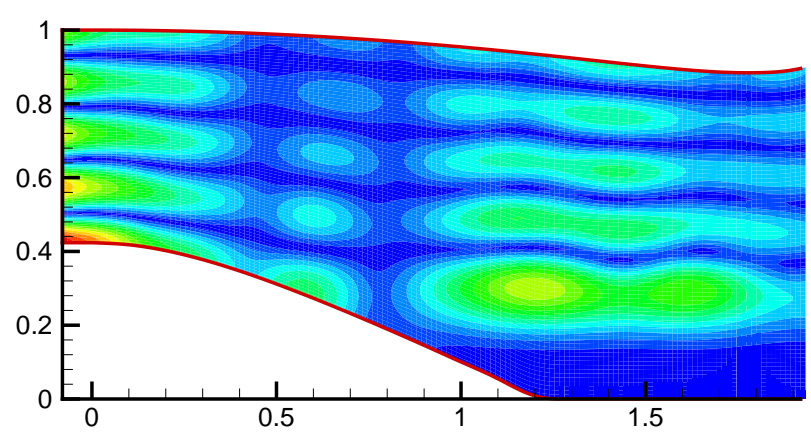

(a) FEM

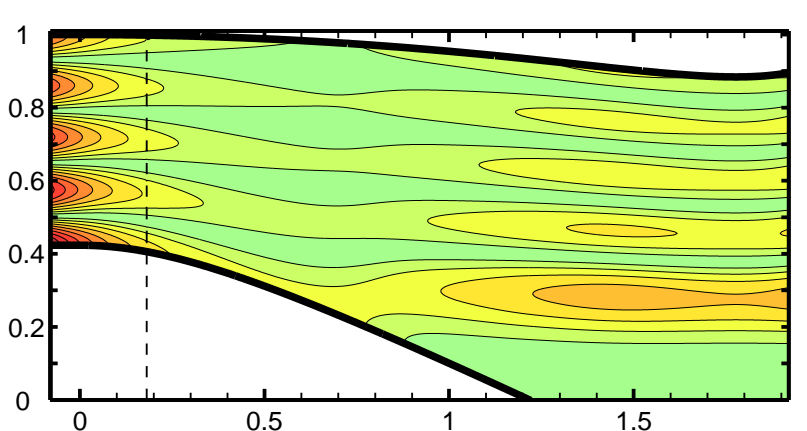

(b) MS

Figure 7. $M=0.5$ with $m=5, \omega=19.8$ and $n=5$. Transition at $x_{t}=0.18$.

Table 7. FEM modal amplitudes and modal power for $M=0.5, m=5, \omega=19.8, n=5$

modal amplitudes (modulus and phase)

modal powers

\begin{tabular}{|c|c|c|c|c|c|c|c|c|}
\hline$n$ & input & \multicolumn{2}{|c|}{ reflected } & \multicolumn{2}{|c|}{ transmitted ${ }^{*}$} & input & reflected & transmitted \\
\hline 1 & 0.00 & $2.67560-03$ & 1.22725 & $3.96519-03$ & 1.94943 & 0.00000 & $2.8561-06$ & $6.6590-07$ \\
\hline 2 & 0.00 & $2.31483-03$ & -3.01568 & $4.52353-03$ & -2.27140 & 0.00000 & $1.6552-06$ & $8.0149-07$ \\
\hline 3 & 0.00 & $9.29487-03$ & -0.79894 & $3.55049-02$ & -1.21018 & 0.00000 & $1.1720-05$ & $3.7076^{-05}$ \\
\hline 4 & 0.00 & $2.40597-02$ & -2.19160 & $1.05487-01$ & 2.57662 & 0.00000 & $4.4124-05$ & $2.3322-04$ \\
\hline 5 & 1.00 & $1.30404-01$ & -0.26518 & $3.69705^{-01}$ & -2.35150 & $0.00000^{\dagger}$ & 0.0000 & $8.4441-04$ \\
\hline 6 & 0.00 & $2.48182-02$ & -0.74233 & $4.75458-03$ & -2.59301 & 0.00000 & 0.0000 & 0.0000 \\
\hline 7 & 0.00 & $3.19080-03$ & -0.60445 & $5.60118-04$ & 0.28489 & 0.00000 & 0.0000 & 0.0000 \\
\hline
\end{tabular}

* Phase of transmitted mode is relative to exit plane.

$\dagger$ The mode is cut-off and therefore propagates no energy on its own, only by interaction with the cut-on reflected modes. 